\title{
Performance Characterisation of Multi- Constellation GNSS-based Positioning for Maritime Applications
}

\author{
Iván Herrera-Pinzón and Stefan Gewies. \\ Department of Nautical Systems, German Aerospace Centre (DLR)
}

\begin{abstract}
BIOGRAPHIES
Iván Herrera-Pinzón is a research fellow at the German Aerospace Centre (DLR), in the Department of Nautical Systems of the Institute of Communications and Navigation. $\mathrm{He}$ is mathematician and geodesist. He received an MSc degree in Geodesy and Geoinformation Science at the Technical University Berlin. Currently PhD candidate in Satellite Navigation and Geodesy, his research focuses on GNSS-based Ground Based Augmentation Systems and associated integrity concepts for maritime purposes.
\end{abstract}

Stefan Gewies is a research fellow at the German Aerospace Centre, in the Department of Nautical Systems of the Institute of Communications and Navigation. He received his $\mathrm{PhD}$ in Physics at the Heidelberg University for his research about modelling of solid oxide fuel cells. Currently, leads the group of Maritime Services, dealing with the development of radio navigation-based services the provision of services for modernised and future GNSS within the maritime context.

\begin{abstract}
Maritime augmentation services are designed to satisfy increased accuracy and integrity requirements on positioning required for safe and efficient vessel's navigation in coastal areas. While currently these services support the usage of code based positioning, increasing accuracy requirements for maritime safety-critical applications demand a detailed analysis of the performance of solutions for multi-constellation based augmentation products, together with their respective integrity information. This paper discusses approaches for alternative multi-constellation GNSS-based positioning techniques through the characterisation of their performance regarding data processing layers of maritime GNSS augmentation services. Feasibility and effectiveness of error characterisation methods are analysed, and special attention is laid on the estimation of usable performance key identifiers for the real-time estimation of the current capabilities of the GNSS-based products, and their possible on-board utilisation.
\end{abstract}

\section{INTRODUCTION}

Reliable and accurate on-board provision of positioning, velocity, and timing (PVT) data is an essential prerequisite for safe and efficient navigation of vessels. Due to their favourable availability, Global Navigation Satellite Systems (GNSS) are the recognised core element for the provision of PVT data for maritime applications. However, the identified vulnerability of GNSS signal propagation effects, as in the case of ionospheric disturbances or jamming, is a challenge to all systems aiming for the resilient provision of PVT data within safety-critical systems.

Therefore, the maritime community developed in the late nineties the IALA Beacon DGNSS service to meet accuracy and integrity requirements for navigation of vessels in coastal areas. However, the development and modernisation of the GNSS constellation grant the opportunity to improve the on-board PVT data provision by exploiting the multiple GNSS signals in multifrequency and multi-constellation approaches. The evolving redundancy in GNSS measurements enhances the accuracy of the PVT data, providing a way to mitigate a large part of the ionosphere threat. It also improves the monitoring of the system integrity by increasing the redundancy of the observations. The increasing performance of GNSS-based positioning is a legitimate reason to examine the future role of maritime GNSS augmentation services.

To demonstrate the benefits that the multi-constellation approach can provide to maritime users in relation to the aforementioned IALA Beacon DGNSS augmentation service, this work discusses the application of both GPS and GLONASS, by the utilisation of linear combinations of single and dual frequency measurements, in maritime applications. Therefore, the objective is twofold: First, the evaluation of positioning performance of these techniques, under maritime conditions in comparison to positioning with the IALA Beacon DGNSS service; and second, the investigation of the error behaviour on these approaches. To provide a test-bed for the experimental validation of these concepts, a multi-sensor architecture supporting the use of multi-frequency and multi-system techniques is provided by the so-called Maritime Ground Based Augmentation System (MGBAS). The MGBAS applies a two-station concept, consisting of a reference station (RS) and an integrity monitoring station (IMS), where the RS is responsible for the provision of corrections and the IMS, acting as a virtual maritime user, is responsible for the evaluation of provided corrections 
and the characterisation of the performance of the multiple positioning solutions.

The evaluation of the real-time performance of the positioning solutions at the IMS station constitutes a key element for the provision of service and data products integrity information, and is expected to contribute to the mitigation of threats during safety-critical applications at the user side. However, the absolute determination of accuracy and integrity of provided augmentation data is often not possible in real-time for the complete service area. An estimation of both can be performed based on performance key identifiers (PKI). This is why, this work introduces a modular concept of error detection and integrity monitoring based on the use of PKI. The fundamental objective is the monitoring of the performance of multi-frequency and multi-constellation GNSS positioning techniques for real-time maritime applications, by using a set of PKI enabling the differentiation between usable/unusable observations, both in nominal conditions and under the presence of perturbations.

\section{MARITIME FUNCTIONAL SERVICE LAYER MODEL FOR GNSS-BASED POSITIONING}

The monitoring of the performance of the GNSS-based positioning is accomplished through the implementation of the generalised model of GNSS augmentation services (IALA, 2015). Service provision and evaluation are realised by four sub-facilities representing the data processing in GNSS Signal Domain, GNSS Position Domain, Service Signal Domain, and Service Position Domain (Figure 1).

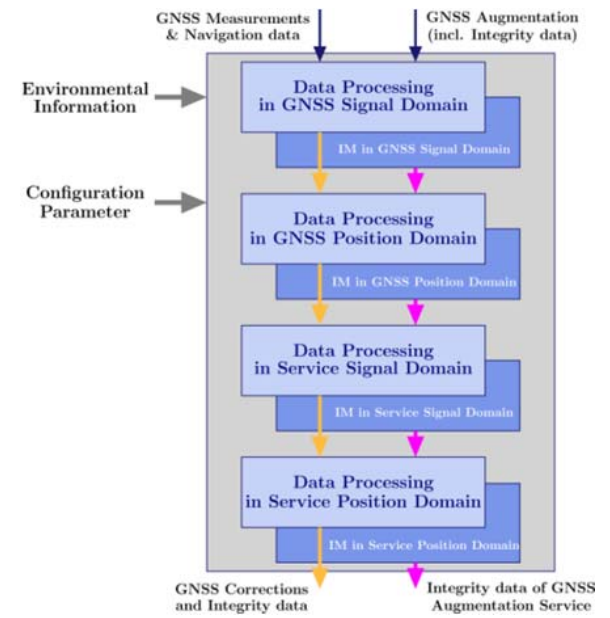

Figure 1: Generalised Data Processing in the GNSS Augmentation Service, based on (IALA, 2015)

Each processing layer is equipped with monitoring functions which contribute to the evaluation of the performance of the GNSS in use, taking into account the different implemented positioning techniques. To overcome the difficulties of the real-time determination of the achieved level of performance at the user site, a set of PKI per sub-facility is defined.

\section{PKI PER FUNCTIONAL SERVICE LAYER}

First, at the GNSS Signal Domain, plausibility and consistency tests are applied to investigate the usability of satellite specific code and carrier phase measurements for GNSS-based positioning. The usability of individual GNSS satellites and their signals for service provision is determined based on the completeness of GNSS range, phase, and navigation data per GNSS satellite in view. In addition, the GNSS Signal Domain applies several plausibility and consistency tests to investigate the usability of satellite specific code and carrier phase measurements for GNSS-based positioning. Applicable performance indicators in this domain are range errors, code and phase noise, carrier-to-noise ratio (CNR), frequency of cycle slips, ionospheric path delays, gradients and multipath. Recent analysis (HerreraPinzón, et al., 2014) have characterised the behaviour of code and phase noise, as well as the CNR, and their relation with positioning errors, but they lack of an estimation of multipath and range errors. Thus, for the scope of this domain, the present work devotes effort on their calculation and determination. Furthermore, it is discussed whether they appropriate indicators to characterise the usability of the GNSS data in the signal domain.

Secondly, at the GNSS Position Domain, the feasibility of GNSS-based positioning (e.g. number of usable satellites, dilution of position), and the achieved reliability (e.g. availability of position and current position error) are verified, taking into account applied positioning techniques. Valid indicators in this domain are the dilution of precision and the number of available (and excluded) satellites, but also the applicability of the outlier detection techniques; in particular Receiver Autonomous Integrity Monitoring with Fault Detection and Exclusion (RAIM-FDE) for the available positioning techniques is also investigated. Thirdly, at the Service Signal Domain, residual errors regarding individual corrections as well as the estimation of decorrelation effects are considered to validate the service provision. In the absence of additional information, applicable performance indicators in this domain are the Pseudorange Residual (PRR) and the User Differential Range Error (UDRE). The performance of these PKI for the IALA Beacon DGNSS service has been already studied by (Gewies, 2015).

Lastly, at the Service Position Domain, the IMS acts as an artificial user to demonstrate the usability of the GNSS augmentation service for positioning and the achievable performance taking into account the supported position techniques. This analysis is based on the number of satellite links for which at IMS own measurements and correction data are available and usable for positioning; the spatial distribution of usable GNSS satellites at the 
IMS; and the accuracy of the positioning at IMS. Applicable indicators in this domain are the positioning errors and their distribution.

\section{MARITIME PORTFOLIO OF GNSS BASED POSITIONING TECHNIQUES}

The increasing number of GNSS in operation -the American Global Positioning System (GPS), the Russian GLObalnaya NAvigatsionnaya Sputnikovaya Sistema (GLONASS), the Chinese BeiDou and the European Galileo- are expected to provide global coverage and accurate positioning in maritime environments. It is expected that a combined usage of the various GNSS signals in multi-frequency and multi-constellation approaches increases the redundancy needed for improved error detection and compensation of GNSS-based PVT determination

Among the many different positioning techniques, the preferred approaches for maritime applications are nowadays the L1-Code based Single Point Positioning (SPP), with achievable horizontal accuracies of a few metres, and the code-based differential GPS (CDGPS) technique, known as IALA Beacon DGNSS, where errors in determination of horizontal positions are reduced to less than $1 \mathrm{~m}$. In DLR's preceding projects, an initial approach for the integrity monitoring of used GPS, applied service components and provided augmentation data was implemented and evaluated within the experimental MGBAS, to work out advantages and disadvantages of an IALA Beacon DGPS like concept of MGBAS error characterisation. However, the redundancy of the shore-side MGBAS was limited by single constellation GNSS-based service provision (Noack, et al., 2009).

To give the experimental proof that the requirements on safety critical applications can be fulfilled by the use of multi-constellation based techniques, this work deals with the probable extension of the portfolio of maritime services. The performance of the following GNSS-based techniques is investigated in the context of maritime applications:

- GPS L1 Single Point Positioning

- GLONASS L1 Single Point Positioning

- Combined L1 Single Point Positioning

- GPS L1/L2 Positioning with Linear Combination

- GLONASS L1/L2 Positioning with Linear Combination

- Combined L1/L2 Positioning with Linear Combination

- Code Differential GPS L1 (CDGPS)

\section{EXPERIMENTAL SETUP}

The implemented concept is realised within the MGBAS at the research port of Rostock, in the north of Germany; where a considerably large amount of daily maritime traffic defines it as a suitable environment for the testing and validation of such a system. Both stations, the RS and the IMS, are constituted by a continuously operating station, recording GNSS data in a time rate of $20 \mathrm{~Hz}$.

All sensor measurements used for the investigations comprise the GPS week 1836, between the $15^{\text {th }}$ and the $21^{\text {st }}$ of March, days of year (doy) 74 to 80 . The behaviour of the proposed performance indicators is investigated with the purpose to detect errors on the observations associated to malfunctions on the system or to natural phenomena disturbing the signal propagation and diminishing the accuracy of the measuremets. The selection of these particular set of data is due to the reported strong ionospheric activity which was registered for the entire week and in particular to the aurora that was observed in the Baltic Sea, close to the Port of Rostock, during the $17^{\text {th }}$ of March (Kieler Nachrichten, 2015).

The analysis of the performance of the implemented system is evaluated using DLR's Real-Time software Framework (RTFramework) implemented in C++ (Gewies, et al., 2012).

\section{RESULTS}

\section{GNSS Signal Domain}

To evaluate the performance of GNSS observables, the sources of errors for each measurement per satellitereceiver link should be investigated. As it was already mentioned, this work focuses additionally on the analysis of the impact of multipath. It is widely accepted that GNSS signals tracked at low elevations result into GNSS observables with decreased data quality due to the increased influence of multipath propagation effects. For improved outlier detection, the nominal behaviour of this error source must be modelled dynamically. Thus, the relation between multipath and elevation angle is analysed to clarify whether an unambiguous dependency holds.

The real-time calculation of the multipath (and receiver noise) for the code observable $\left(M P_{i}\right)$ is performed using the following simplified measurement model (Borre \& Strang, 2012):

$$
\begin{aligned}
& M P_{1}=P_{1}-\left(\frac{2}{\alpha-1}+1\right) \varphi_{1}+\frac{2}{\alpha-1} \varphi_{2} \\
& M P_{2}=P_{2}-\frac{2 \alpha}{\alpha-1} \varphi_{1}+\left(\frac{2}{\alpha-1}-1\right) \varphi_{2}
\end{aligned}
$$

with $P_{i}$ as the code measurement on $L_{i}, \varphi_{i}$ the phase measurement on $L_{i}$, and $\alpha=\left(\frac{f_{1}}{f_{2}}\right)^{2}$, the squared ratio between the frequencies of the $L_{1}$ and $L_{2}$ signals. For this derivation two important assumptions hold: first, $\mathrm{MP}_{\mathrm{i}}$ is assumed to be zero-mean over long intervals of observations, and second, it is assumed that phase noise 
plus multipath is small in comparison with pseudorange noise plus multipath and therefore can be neglected.

Thus, Figure 2 shows the behaviour for the estimated L1 code multipath $\left(\mathrm{MP}_{1}\right)$ for satellite GPS 12 (blue), in relation to its elevation angle (green), for the data collected at the IMS, over two days of the time interval of this study: doy 74 and 76. The calculated multipath displays the well-known spatial dependency with respect to the elevation angle, with the highest values for the variable at low elevation angles (see Figure 2, at doy $74.25,74.62,74.88$ for daily maxima), and the lowest values for the multipath associated to high elevation angles (see Figure 2, at doy 74.75 and 76.75 for daily minima).

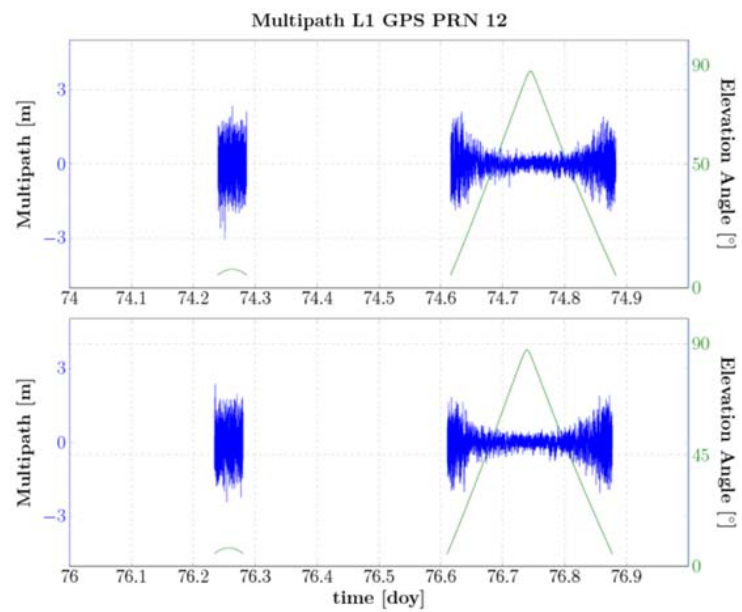

Figure 2: Multipath (+noise) MP1 (blue), and elevation angle (green) over time, for two selected days of GPS PRN 12

Moreover, a detailed analysis of the temporal behaviour of the multipath (Figure 2) shows a marginal shift of maxima to earlier hours for following days. Such changes are associated to the shift due to the repetition of the geometry (each sidereal day). Lastly the assumption of a zero-mean behaviour for the multipath holds for the entire data set. To have a closer look on the behaviour of the multipath, Figure 3 shows the summary of statistics for the same satellite, disaggregated by day. Minimum daily values range from -2.9 to $-2.2 \mathrm{~m}$, with the smallest value obtained for doy 76. Maximum values are in the range of 2.0 to $2.6 \mathrm{~m}$, exhibiting the largest value again for the same doy. The standard deviation of the multipath is nearly the same for every day, although doy 76 outstands again with the largest value $(0.53 \mathrm{~m})$.

Figure 3 displays additionally the analysis results for the entire GPS system. Comparing maxima, minima, and standard deviation of the single satellite and the entire system it follows that GPS PRN 12 shows typical multipath values for GPS satellites. However, during doy 76 and 80 the extreme values of the multipath for this satellite drive the behaviour of the extreme values of the whole constellation.

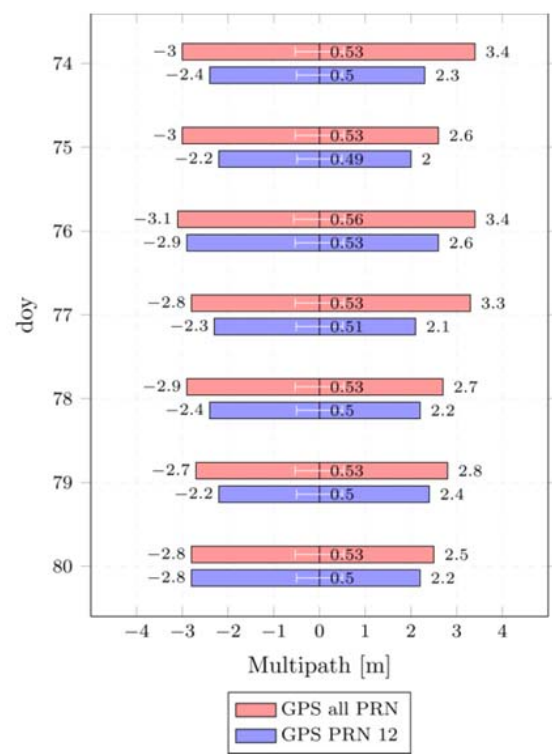

Figure 3: Daily multipath (+noise) MP1 for GPS PRN 12 (blue) and the entire GPS system (red). Labels indicate minima, maxima and standard deviations

Calculated in the same way, Figure 4 shows the behaviour for the L1 code multipath $\left(\mathrm{MP}_{1}\right)$ for satellite GLONASS 10 (blue), in relation to the elevation angle (green), collected during the same time interval at the IMS, and displayed for the two days of interest. Anew, the expected functional relation between multipath and elevation angle is observed along the whole time of the study. The temporal variation of the variable is not observed as easily as in the aforementioned GPS PRN 12, as the repetition of the geometry for the GLONASS case occurs every sidereal week.

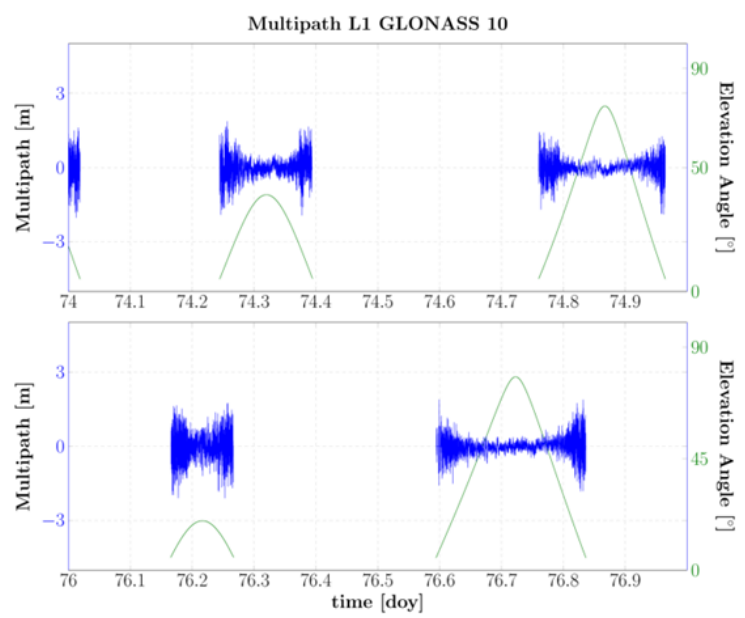

Figure 4: Multipath (+noise) MP1 (blue), and elevation angle (green) over time, for two selected days of GLONASS 10

The behaviour of the multipath for this satellite is provided in Figure 5, detailed by day. Minimum values range between -2.5 and $-2.0 \mathrm{~m}$, where the smallest value is found on doy 74 and 75 . Maximum values range between 1.9 and $2.1 \mathrm{~m}$, with the largest value during doy 
77 and 79. Finally, the standard deviation takes values between 0.38 and $0.45 \mathrm{~m}$, with maximum value during doy 79. The overall behaviour of the multipath for the entire GLONASS constellation along the complete time of study is also displayed in Figure 5, disaggregated by day, where a rather homogenous behaviour - particularly regarding the standard deviation- is observed for each day. Functional dependencies with other variables must be examined for the adequate modelling of multipath in GLONASS satellites.

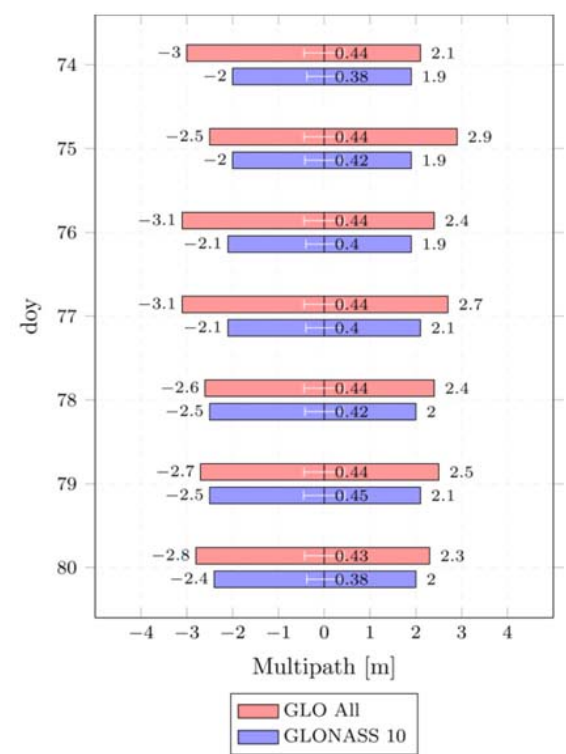

Figure 5: Daily multipath (+noise) MP1 for GLONASS 10 (blue) and the entire GLONASS system (red). Labels indicate minima, maxima and standard deviations

The second relevant source of error of the measurements on the GNSS Signal Domain is the ionospheric delay. Ionospheric disturbances affect the velocity of GNSS signals, changing their apparent ranges to satellites. The shift in range due to this effect is called ionospheric delay. It is frequency dependent affecting signals in different amounts, and increases with low elevation angles. To reduce the influence of the elevation angles, the rate of change of the ionospheric delay is additionally calculated.

Figure 6 shows the time series of the rate of change of the ionospheric delay (blue) for GPS satellite PRN 12, in $\mathrm{m} / \mathrm{min}$, over the two particular days of this study: doy 74 and 76. A clear dependency with the elevation angle is again observed, where extreme values are (generally) associated to low elevation angles. The behaviour of doy 74 is rather stable, with no evident outliers. However, data for doy 76 displays an anomalous behaviour from ca. 76.65 to 76.80 , where although the magnitude of the obtained values does not surpass considerably the daily minima or maxima (-4.0 and $4.0 \mathrm{~m}$, respectively), an irregular distribution is observed. Since the occurrence of these events is sporadic and their magnitudes are within the tolerance of the daily behaviour, an analysis of the statistics of these events in comparison with the overall (daily/weekly) behaviour does not provide additional information for identification of the disturbances. Therefore, the analysis of the time series is preferred.

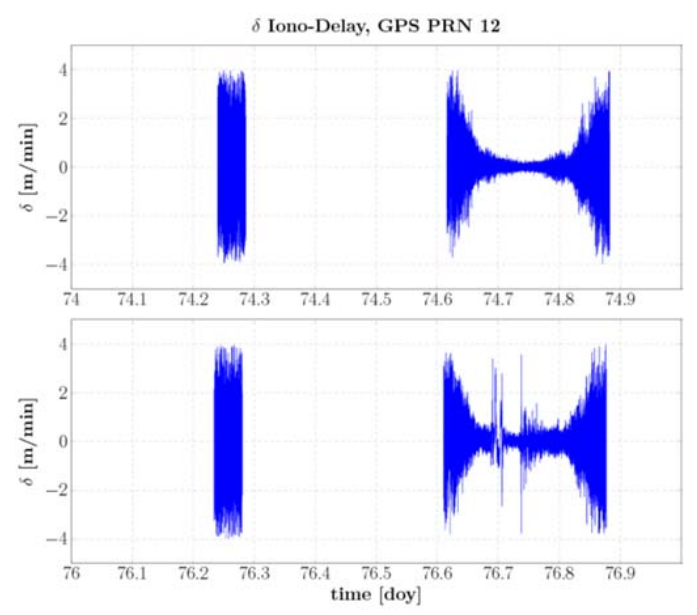

Figure 6: Time rate of ionospheric delay, for selected days. GPS PRN 12

In a similar way, Figure 7 shows the time series of the rate of change of the ionospheric delay for GLONASS satellite 10 (blue), in $\mathrm{m} / \mathrm{min}$, over the two days of study. Data for doy 74 shows a steady relation between the elevation angles and the rate of ionospheric delay (see Figure 4), while for doy 76 an anomalous behaviour is once again observed at ca. 76.7 .

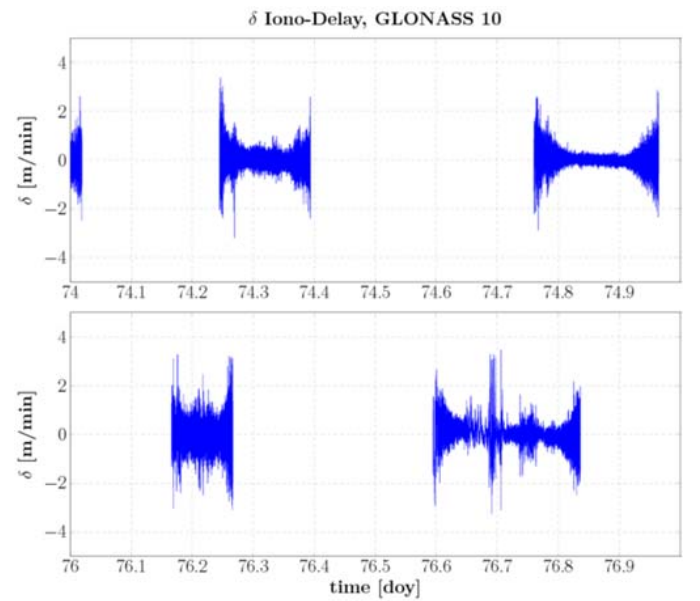

Figure 7: Time rate of Ionospheric Delay, for selected days. GLONASS 10

These anomalous behaviours displayed during doy 76 , particularly on the interval $(76.6,76.8)$, confirm the reports of occurrence of a strong located ionospheric activity over the area of study. The impact of these phenomena on the quality of the measurements is expected to degrade the quality of solutions during the positioning determination process; therefore their behaviour has to be further considered and mitigated.

The last part of the analysis in this domain covers the estimation of the residual errors per satellite as an 
indicator to analyse the expected range quality for positioning solutions. Figure 8 depicts the time series of residual errors for the $\mathrm{L} 1$ code observations of satellite GPS PRN 12, for the two selected days. These time series indicate that the residual errors are largely dominated by the influence of multipath, thus following the same functional dependency with respect to the elevation angle which was seen in Figure 2.

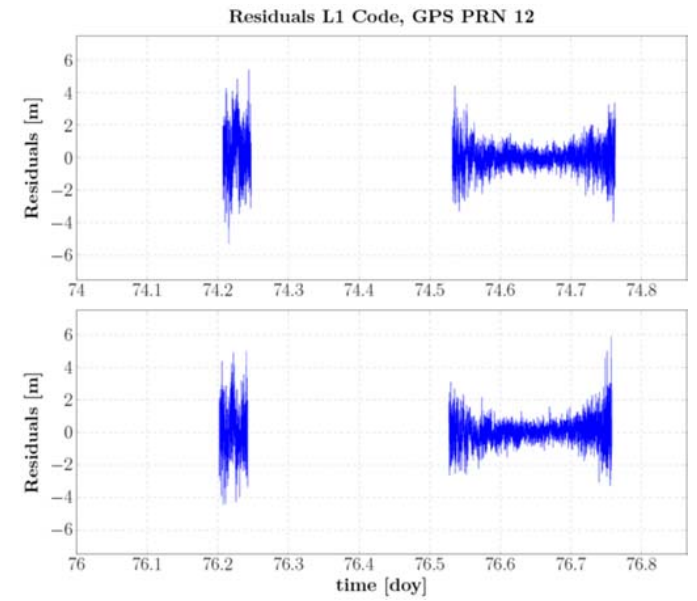

Figure 8: GPS L1 code residuals (blue), for two selected days, of PRN 12

For a closer look into the behaviour of the GPS L1 code residuals, Figure 9 summarises its behaviour for each day of observations. Extreme values for minima and maxima are found in doy $78(-6.2 \mathrm{~m})$ and doy $76(7.6 \mathrm{~m})$, respectively. However, for PRN 12 standard deviations are closely similar during the entire week. The behaviour of the residuals for this satellite with respect to the overall daily constellation can be derived from Figure 9. An inspection to these values shows that PRN 12 has a nominal behaviour for maxima and minima within the days of study. It is worth to notice that the extreme values for this variable are obtained during doy 76 and 78. The overall behaviour of the GPS constellation and individual satellites, such as the PRN 12, reinforce the idea that these days must be looked carefully, as potential irregularities may decrease the performance of individual links impacting negatively the final accuracy of positioning techniques.

Similarly, Figure 10 shows the time series of residual errors for the L1 code observations of satellite GLONASS 10 , for the proposed days of study. It is noticeable that the behaviour of the residual errors are largely dominated by the presence of multipath, although results indicate a larger intra-variation as the time series exhibit a much nosier behaviour, particularly in those sections with low elevation angles. A notable variation is observed at doy 76.55 , where the distribution of this indicator is rapidly altered, surpassing the nominal behaviour.

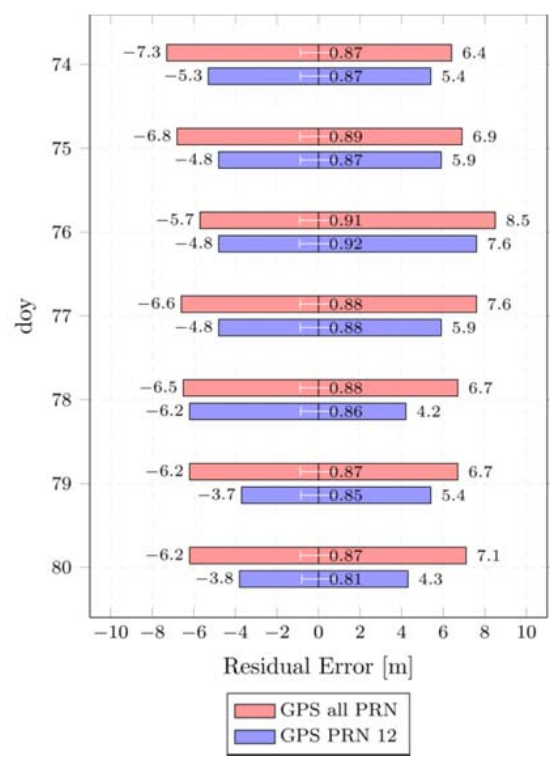

Figure 9: Daily code $L 1$ residual errors for GPS PRN 12 (blue) and the entire GPS system (red). Labels indicate minima, maxima and standard deviations

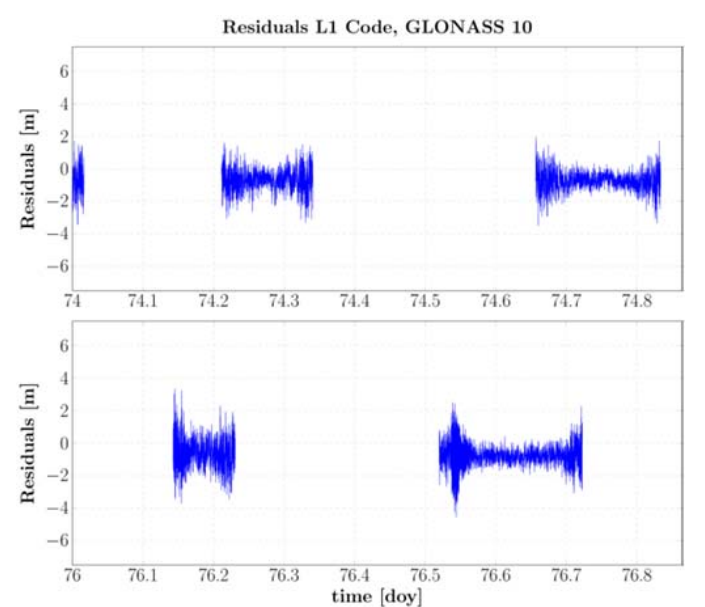

Figure 10: GLONASS L1 code residuals (blue), for two selected days, of satellite 10

Figure 11 provides a closer look to these results, with the daily behaviour of the residual errors for this satellite. Minima and maxima values, -4.7 and $6.0 \mathrm{~m}$, are obtained at doy 78. Standard deviation for the variable varies between 0.64 and $0.84 \mathrm{~m}$.

For a full perspective of the behaviour of the residual noise, Figure 11 shows additionally the performance for the entire GLONASS constellation during the time of the study. It is clear that satellite 10 exhibits a fairly good performance in comparison to the extreme values displayed by the whole constellation. Of special interest are the largest (negative) error values observed during doy 75 to $77(-16.7,-14.3$ and $-13.1 \mathrm{~m})$, which are indicator of potential threats for positioning techniques. This behaviour, characterised for large magnitudes of residual errors, may lead to failures and inaccuracy during the positioning process. 


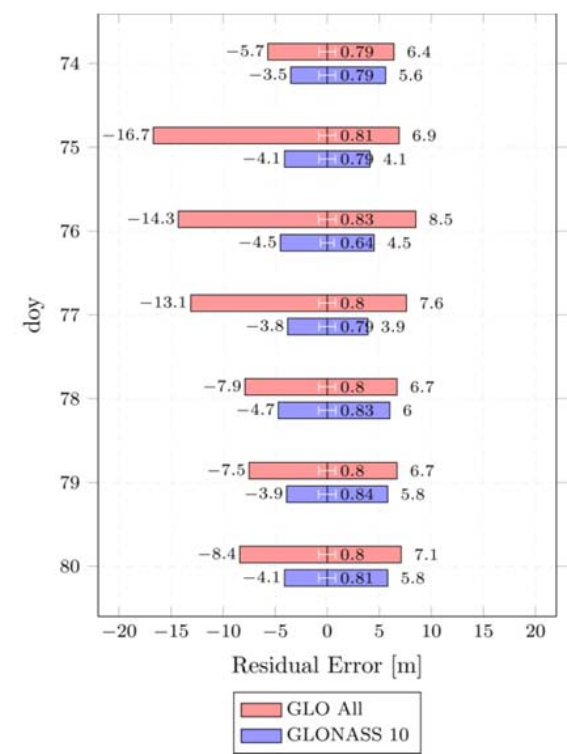

Figure 11: Daily code L1 residual errors for GLONASS 10 (blue) and the entire GLONASS system (red). Labels indicate minima, maxima and standard deviations

Early stage processing levels at the GNSS Signal Domain, are therefore a valuable tool which provides information regarding error characterisation on individual links satellite-receiver and indicates the usability of the collected measurements. Multipath and residual errors estimated within the MGBAS can be used as an alternative to leverage the measurements in posterior processing processes, via stochastic models. An accurate quantification of the error in this domain, in relation with the overall nominal behaviour provides valuable tools for the early detection and exclusion of potential outlier observations.

\section{GNSS Positioning Domain}

It is known that a poor geometry constellation and poor satellite availability derive in an increased error on the final positioning solution. Furthermore, site specific conditions, such as obstacles or effects affecting the individual links to the satellites (multipath and non-lineof-sight) may decrease the signal availability impairing the Dilution of Precision (DOP) and ultimately the performance of positioning. DOP values are considered as an estimate to describe the influence of constellation geometry on positioning and timing. Different DOP designations are available, e.g. HDOP for horizontal positioning or TDOP for timing. Epochs with a HDOP smaller than 7.5 are considered appropriated for position determination (Borre \& Strang, 2012). The adequacy of DOP constitutes a performance indicator for positioning and requires an appropriate spatiotemporal modelling and understanding of its behaviour.

Although the IMS is located at a mid-latitude (ca. $54^{\circ} \mathrm{N}$ ), the HDOP is in most of the cases smaller for the GPS case than for the GLONASS case. Albeit this difference is not considerably large, this may indicate that the quality of solutions derived of GPS-only processing may have marginal better HDOP that those derived with GLONASS-only techniques. As expected, the combined usage of GPS and GLONASS delivers the best values for this indicator, and indication of the potential advantages of the usage of a combined solution. The use of more constellations provide benefits in terms of the geometric distribution and is expected to be associated to the provision of more accurate (smaller) protection levels and therefore improving the performance of the GNSS based positioning. The result of this analysis confirms that a HDOP threshold of 7.5 for safe solutions needs to be revalidated.

For the scope of this study, the analyses of the HDOP for three different geometries are of interest: a GPS-only constellation, a GLONASS-only and the combined GPS/GLONASS constellation. Figure 12 shows the behaviour of the HDOP for doy 74 and 76 at the IMS, where the green line represents the results obtain for GPS, the blue line for GLONASS, and the red line for the combined constellation. The entire data set displays fairly good HDOP values for the three configurations, as they account the excellent visibility conditions for the reception of GNSS signals at the IMS. Values of the HDOP during the observed epochs are around 1 for GPSonly and GLONASS-only and are distinctly lower using GPS and GLONASS, below the suggested safe threshold with only few and small changes due to sudden variations in the number of satellites used for positioning.

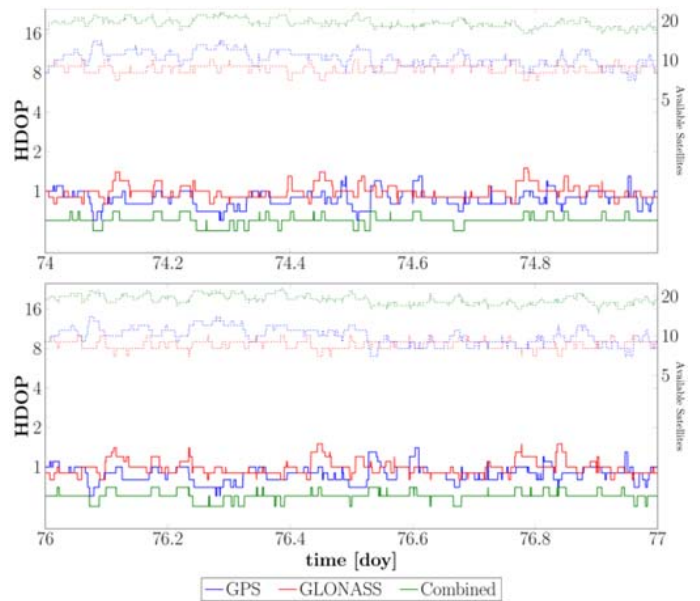

Figure 12: Time series of HDOP (solid lines) in relation to the number of satellites (dotted lines) for the three constellations

The second part of the analysis in this domain deals with the applicability and benefits of fault detection and exclusion techniques. The estimation of a 3D GNSSbased position requires four satellites. When the provision of a mechanism to detect unreliable satellites is intended, at least 5 satellites are demanded. If the exclusion of unreliable satellites is intended, a minimum of 6 satellites are necessary. RAIM algorithms are used to identify failures on satellites by examining all position solutions 
obtained with various subsets of the available ranging measurements. The detection process relies on a chisquared test of the test statistic calculated as the sum of squared least-squares residuals. For the exclusion of the inconsistent measurement, the creation of subsets excluding one satellite, calculation of their corresponding test statistics and exclusion based on these test statistics is performed. Results of the exclusion process are considered as an indicator of the usability of the satellite.

In this context, a RAIM analysis was performed with the purpose to identify potential threats for positioning while using individual observations. For this analysis a frequency of $1 \mathrm{~Hz}$ of data was used. Table 1 summarises the number of epochs where the RAIM algorithm has advised the exclusion of particular GPS satellites. Results exhibit a rather anomalous behaviour for doy 76 in comparison with the overall week, where the number of individual epochs with one or more excluded satellites comes to 7516 .

Table 1: Number of epochs with excluded GPS satellites, according to RAIM

\begin{tabular}{|c|c|c|c|c|c|c|c|}
\hline doy & $\mathbf{7 4}$ & $\mathbf{7 5}$ & $\mathbf{7 6}$ & $\mathbf{7 7}$ & $\mathbf{7 8}$ & $\mathbf{7 9}$ & $\mathbf{8 0}$ \\
\hline \#Epochs & 21 & 19 & 7516 & 47 & 107 & 5 & 25 \\
\hline
\end{tabular}

For a better look to this characteristic behaviour, Figure 13 displays the time series for the number of excluded satellites for doy 76. Time intervals at doy 76.35 to 76.55 and 76.6 to 76.8 are responsible for the majority of epochs with satellite exclusions. At the first time interval the number of excluded GPS satellites starts unsteady with one and increases at around 76.5 up to 3 satellites whereas in the second interval it only shortly increases up to 2 satellites. These anomalous behaviours correlate in time with the calculated ionospheric activities for this day (Figure 6 and Figure 7). These time intervals are expected to display the worse indicators in other domains and must be observed carefully.

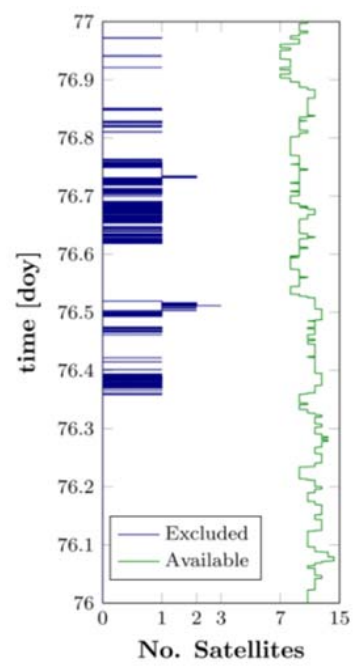

Figure 13: Time series of excluded GPS satellites (blue), according to RAIM, for doy 76. Available satellites are additionally displayed (green)

\section{GNSS Service Domain}

At the service positioning domain, the IMS acts as an artificial user to validate the feasibility and the performance of positioning taking into account the applied positioning techniques and RAIM-FDE. The evaluation of position accuracy is performed by comparing the determined positions with respect to the surveyed value of the user station. The difference between both is the horizontal positioning error (HPE).

The first technique to be considered is the single point positioning service. Figure 14 shows the time series of HPE for the GPS L1 single point positioning -as standard technique for vessels navigation- during the two selected days of interest, where the HPE is below $3 \mathrm{~m}$ with the exception of doy 76.4 to 76.5 and 76.6 to 76.7 . Within this time intervals the HPE remains above the $3 \mathrm{~m}$ for more than one hour. These results correlate the aforementioned findings of the RAIM technique. Additionally, Figure 21 summarises the overall behaviour of this technique for the entire week. The general performance of the technique is fairly acceptable, with mean HPE values between 1.0 and $1.6 \mathrm{~m}$, reaching its maxima at doy 76 ; maximum values for the HPE ranging between 4.8 and $6.6 \mathrm{~m}$, with a maxima on doy 76; and an almost uniform standard deviation of ca. $0.7 \mathrm{~m}$, except for doy $76(1.3 \mathrm{~m})$. The high variance and the presence of extreme values of HPE for doy 76 are consistent in time with the ionospheric activity reported for these days.

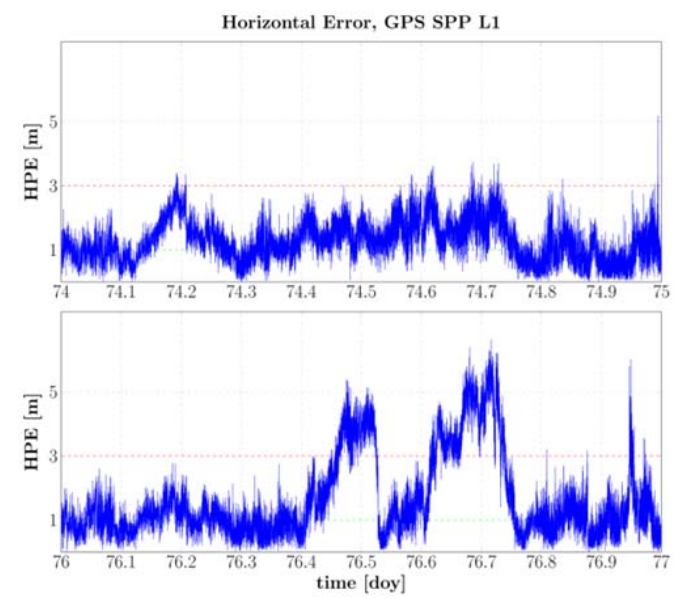

Figure 14: Horizontal positioning error for GPS L1 SPP. Green and red lines indicate accuracy levels of 1 and $3 \mathrm{~m}$, respectively

A further step in the analysis of the available techniques is made through the analysis of the HPE for a GLONASS SPP L1 solution (Figure 15). A rather more unstable behaviour on the indicator is observed. The high variability on the indicator is consistent with the drops on the HDOP reported in the previous section (cf. Figure 12). The impact of the geometry on the positioning solution is considerably large. The results displayed in Figure 15, in addition to the overall behaviour seen is Figure 21, indicate that a GLONASS SPP L1 solution is not be 
acceptable for most of the safety critical applications. However, as mean values for the HPE are below the $3 \mathrm{~m}$ (Figure 21), these services can be used during low accuracy (coastal) operations. In spite of the low performance in term of accuracy, it is also evident from the time series in Figure 15 an increased variation of HPE for doy in relation, likely related to the aforementioned ionospheric disturbance in this day.

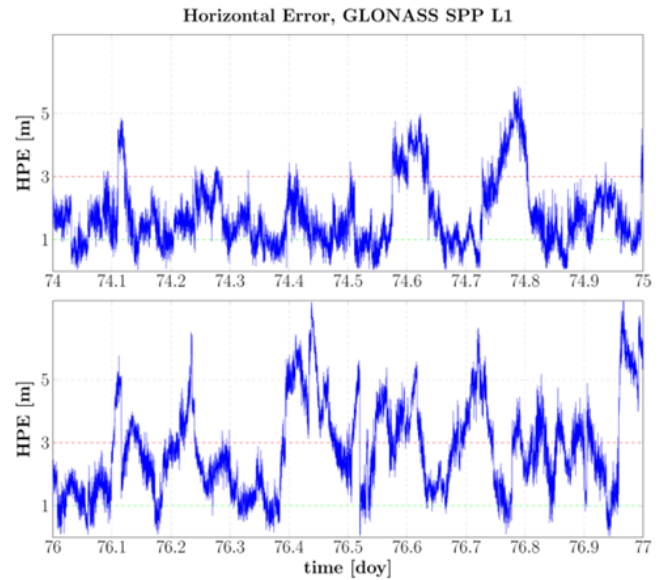

Figure 15: Horizontal positioning error for GLONASS L1 SPP. Green and red lines indicate accuracy level of 1 and $3 \mathrm{~m}$, respectively

The results of the SPP using GPS and GLONASS L1 signals is displayed in Figure 16, for the two selected days. Similar to the performance obtain with GPS-only solution, the results of this technique display in general a favourably behaviour, with a considerable reduction of the maximum daily HPE (Figure 21), and smooth improvements in the values of both the mean and the standard deviation of the HPE. Despite the improvement of the combined solution compared to the GPS-only one, the influence of the ionospheric disturbances is still evident, particularly for doy 76 and 78, where the maximum values for the mean HPE can be found.

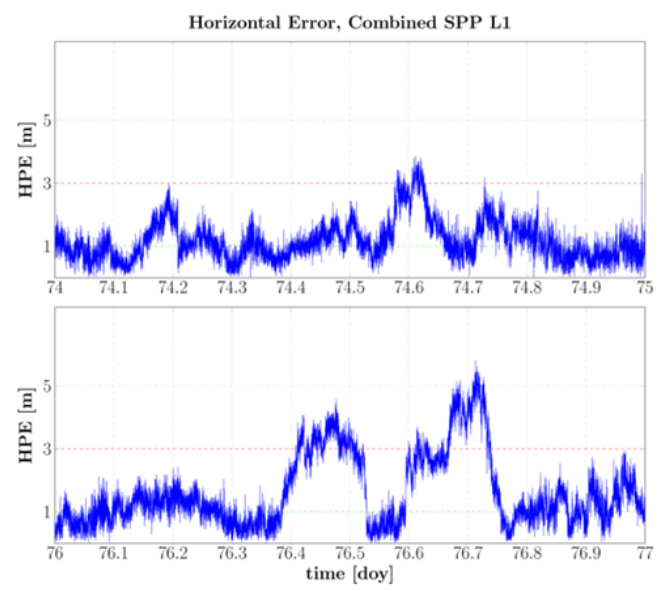

Figure 16: Horizontal positioning error for a combined L1 SPP. Green and red lines indicate accuracy levels of 1 and $3 \mathrm{~m}$, respectively
The next technique is the aforementioned CDGPS L1, which constitutes the current standard for coastal operation in maritime navigation. Figure 17 shows the temporal behaviour of the HPE obtained for the two days of interest, using the mentioned code differential technique. Performance of the indicator is generally acceptable, bounded by $3 \mathrm{~m}$ but in most cases better than $1 \mathrm{~m}$, with an overall noisy solution. However several time intervals are affected by small disturbances that are not noticed during the analysis of the SPP solutions. Decorrelation effects, such as the age of the code corrections, the distance between the RS, and the user differential range error reported for the corrections (Gewies, 2015), are the possible causes for this abnormal behaviour.

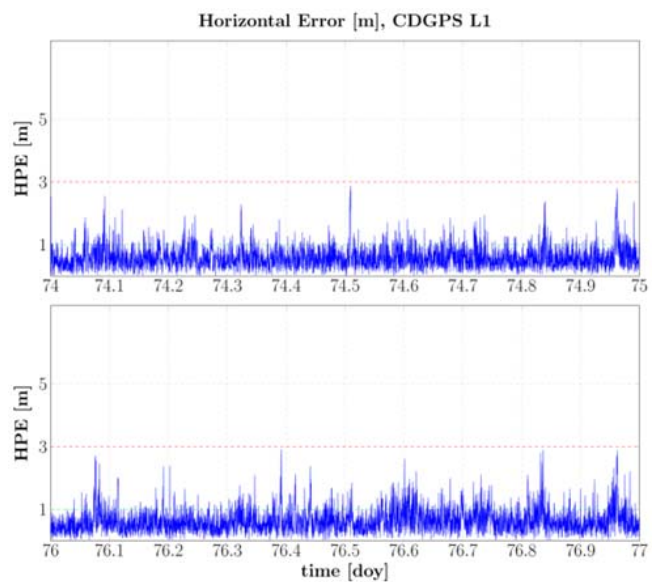

Figure 17: Horizontal positioning error for CDGPS L1. Green and red lines indicate accuracy levels of 1 and $3 \mathrm{~m}$, respectively

Figure 21 provides a detailed view of the statistical analysis of these results. A general improvement in the mean value of the HPE with respect to the values of the SPP technique is achieved. Mean values of ca. $0.60 \mathrm{~m}$ with standard deviations of ca. $0.45 \mathrm{~m}$ suggest that this technique provide better results, in nominal cases, than its SPP counterpart. The expected influence of the ionosphere on doy 76 and 78 is also missing in Figure 17, as the code corrections used by this technique are expected to contain an account for the ionospheric error budget (Borre \& Strang, 2012).

The last technique to be evaluated through the HPE as $\mathrm{PKI}$ is the L1/L2 positioning using linear combination. In the first place, this linear combination is used for a GPS solution. Figure 18 displays the time series for this indicator, with a temporal behaviour similar to those obtained while using GPS SPP L1. Remnants of the aforementioned ionospheric disturbances are also visible in these solutions, although their magnitude is considerable smaller. 


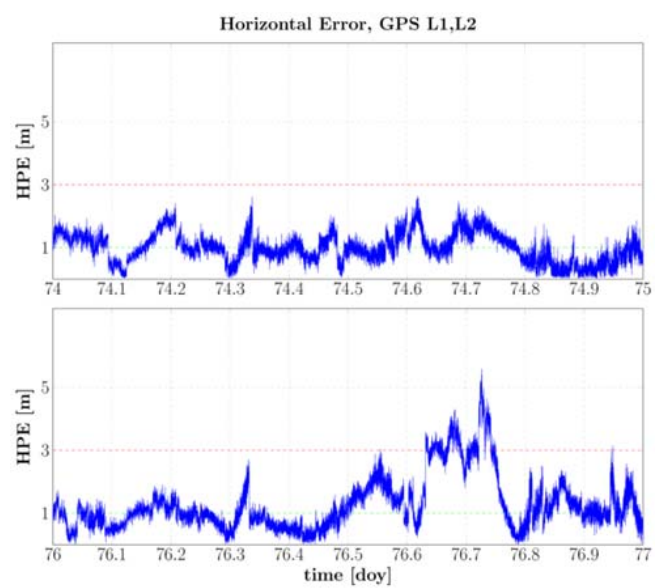

Figure 18: Horizontal positioning error for GPS linear combination. Green and red lines indicate accuracy levels of 1 and $3 \mathrm{~m}$, respectively

For a closer inspection to the behaviour of this service, Figure 21 summarises the main parameters of these results. Compared to the results obtain with GPS SPP L1 measurements, the solution of these technique show a significant reduction of the mean values of the HPE, with almost the whole set of data below the threshold of $1 \mathrm{~m}$ and reaching the $0.8 \mathrm{~m}$ in the best cases. Once again, the maxima of the HPE is found on doy 76, but its magnitude is largely reduced in comparison with the values obtain for the GPS SPP L1. Large reductions on the maximum values of the HPE are also noticeable for the entire data set, where most of the values are improved in several metres. Small but steady improvements on the values for the standard deviation are also evident. The comparison of these results with respect to those obtained with the CDGNSS technique provides also favourable conclusions. In general terms, the mean values and standard deviations of HPE obtained with the former technique are not quite distant form those derived of the latter, with only differences of $0.20 \mathrm{~m}$ in the mean values. Although remnants of the influence of the ionosphere are still notorious on these solutions, the noticeable reduction of the magnitude of maximum errors, as well as the favourable mean HPE values provide sufficient evidence to support the utilisation of these technique in multiple maritime applications.

Similarly, Figure 19 displays the solutions obtained for the point positioning with ionosphere-free linear combination in relation to only GLONASS observations. The improvement with respect to the GLONASS L1 SPP is once again evident. However, the great variability observed on the former technique is still present on the latter, accounting the large influence of the geometric distribution of the constellation into the final solutions. Figure 21 provides an overview of the behaviour of this technique. The improvement on the mean values of HPE with respect to sole GLONASS SPP L1 is evident; with positive changes of up to $0.40 \mathrm{~m}$. Maximum values of HPE are generally reduced. Although the use of the linear combination improves the performance of the solutions when compared with a GLONASS SPP L1 technique, their results continue being inferior to those obtained with the CDGPS technique.

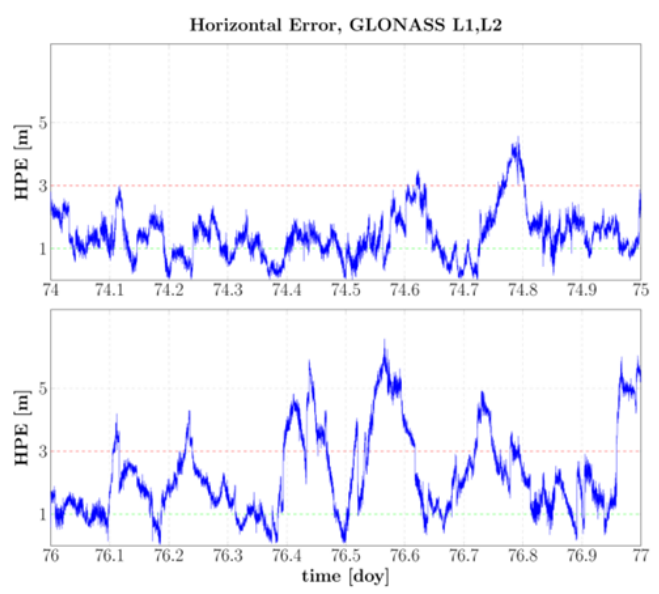

Figure 19: Horizontal positioning error for GLONASS linear combination. Green and red lines indicate accuracy levels of 1 and $3 \mathrm{~m}$, respectively

The last step in this study is the analysis of a combined GPS/GLONASS solution with observables derived from the ionosphere-free linear combination. For this, Figure 20 displays the time series of the obtained HPE. With a temporal behaviour dominated by the solution derived of the GPS ionosphere-free linear combination technique, the overall behaviour is quite steady without the presence of significant outliers. A closer look to this result is presented at Figure 21. An evident improvement on the maximum values of HPE with respect to the GPS with ionosphere-free linear combination is achieved, although the mean and standard deviation values remain almost unaltered. With mean values of HPE close to the threshold of $1 \mathrm{~m}$ and maximum HPE bounded with the value of $3 \mathrm{~m}$, with the exception of doy 76 where the remnants of the ionosphere are still present, this solution stands as a plausible alternative to the use of the CDGPS technique.

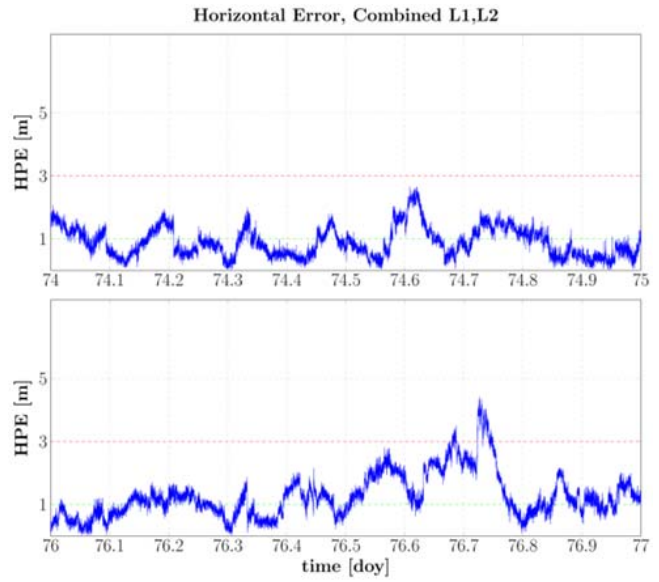

Figure 20: Horizontal positioning error for combined linear combination. Green and red lines indicate accuracy levels of 1 and $3 \mathrm{~m}$, respectively 


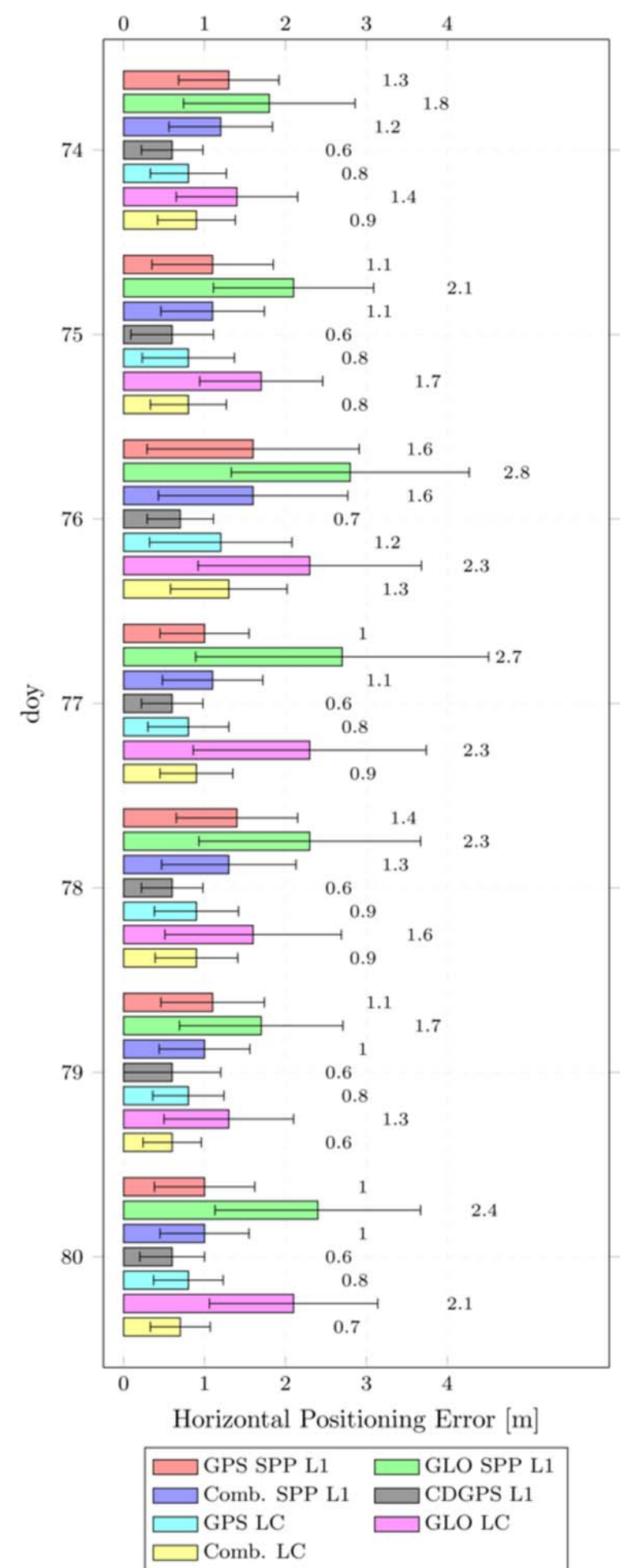

Figure 21: Performance of the Portfolio of Maritime Services. Mean values (in labels) and standard deviations of horizontal positioning errors

\section{SUMMARY AND OUTLOOK}

The performance evaluation of multi-constellation based positioning techniques using the levels of analysis of the generalised model of GNSS augmentation services is presented. This methodology has proven to provide not only valuable information about the current capabilities of the GNSS involved, but also the suitability of different positioning techniques towards maritime applications.

Different PKI have been explored to characterise the performance of individual satellites signals. The so-called GNSS Signal Domain proposes the calculation of the multipath and residual errors for the initial characterisation of the errors on individual links satellitereceiver. The analysis of these parameters may lead to the early exclusion of outlier observation, as well as they provide a useful way to determine the leverage of the measurements. The analysis results of the PKI described in the GNSS Positioning Domain illustrates the advantages of the applicability of RAIM-FDE techniques. The identification of epochs with satellites presenting an anomalous behaviour result of great interest as it enhances the accuracy of the final solutions and contributes to the mitigation of threats, as it was shown for the ionospheric disturbances.

Notable results of this analysis are those obtained on the GNSS Service Domain. Horizontal positioning errors are used at this domain to characterise the capabilities of different positioning techniques and augmentation products. The use of multi-constellation and multifrequency approaches has shown significant improvements with respect to the traditional GPS L1 SPP used within the context of maritime navigation. The proposed approaches use a combination of GPS and GLONASS constellation, followed by a combination of signal with the so-called ionospheric-free linear combination. Particularly, the GPS/GLONASS approach is proven to provide the necessary levels of accuracy for several maritime operations. Their indicators regarding the maximum, mean and standard deviation of the HPE exhibit performances similar in magnitude to those of the CDGPS technique and are clearly superior to those obtained for the GPS L1 technique, with the additional advantage that these proposed techniques do not rely on the acquisition of external correction data. Moreover, the use of multi-frequency approaches has shown additional advantages towards the mitigation of threats during irregular ionospheric activities.

Future activities include the study and modelling of these PKI over longer time series, to determine interdependencies among them and to determine their seasonal variations; the study of outlier detection methods alternative to RAIM, to enhance the robustness of the detection of failures, and the experimentation with additional positioning techniques, such as the Real Time Kinematic and the Precise Point Positioning.

\section{ACKNOWLEDGMENTS}

The authors gratefully acknowledge the contributions and helpful insights of their colleague Dr. Evelin Engler, 
without which the present study could not have been completed.

\section{REFERENCES}

Borre, K., Strang, G. (2012), Algorithms for Global Positioning, Massachusetts, USA, Wellesley-Cambridge Press, ISBN 978-0-980-23273-8.

Gewies, S., Becker, C., Noack, T. (2012), Deterministic Framework for parallel real-time Processing in GNSS Applications, 6th ESA Workshop on Satellite Navigation Technologies (Navitec), Noordwijk, The Netherlands, European Space Agency (ESA).

Gewies, S. (2015), Statistical Behavior of UDRE provided by IALA Beacon Stations at Baltic Sea, Marine Traffic Engineering Conference and International Symposium Information on Ships. Kołobrzeg, Poland. Maritime University of Szczecin

Herrera-Pinzón, I., Born, A., Engler, E. (2014), A Study on Integrity Monitoring for a Multi-Sensor/MultiFrequency GNSS-Based Maritime Ground Augmentation System, Proceedings of the 7th ESA Workshop on Satellite Navigation Technologies (NAVITEC), Noordwijk, The Netherlands, European Space Agency (ESA).

Herrera-Pinzón, I., Born, A., Engler, E. (2015), Modelling of Performance Key Identifiers for Characterisation of Current Capabilities on Maritime Augmentation Systems, Proceedings of the International Symposium on Certification of GNSS Systems \& Services (CERGAL), Darmstadt, Germany, German Institute of Navigation (DGON).

IALA (2015). Performance and Monitoring of a DGNSS Service in the Band 283.5-325 KHz. Guideline 1112, Edition 1, International Association of Marine Aids to Navigation and Lighthouse Authorities.

Kieler Nachrichten (2015), March $18^{\text {th }}$, Polarlicht über der Ostsee. Online Edition. Sect. Bilder Bilder: Kiel Fotostrecke: Polarlicht 17.03.2015

Noack, T., Engler, E., Klisch, A., Minkwitz, D. (2009), Integrity Concepts for Future Maritime Ground Based Augmentation Systems. Proceedings of the 2nd GNSS Vulnerabilities and Solutions Conference, Baska, Croatia, Royal Institute of Navigation.

Teunissen P., Kleusberg, A. (1998), GPS for Geodesy. Walter de Gruyter, 2nd completely revised and extended edition, ISBN: 3-540-63661-7. 